\title{
William's syndrome: gene expression is related to parental origin and regional coordinate control
}

\author{
Jeremy C Collette ${ }^{1}$, Xiao-Ning Chen ${ }^{1}$, Debra L Mills ${ }^{2}$, Albert M Galaburda ${ }^{3}$, Allan L Reiss ${ }^{4}$, Ursula Bellugi ${ }^{5}$ \\ and Julie R Korenberg ${ }^{1,6}$
}

\begin{abstract}
William's syndrome (WS) features a spectrum of neurocognitive and behavioral abnormalities due to a rare $1.5 \mathrm{MB}$ deletion that includes about 24-28 genes on chromosome band 7q11.23. Study of the expression of these genes from the single normal copy provides an opportunity to elucidate the genetic and epigenetic controls on these genes as well as their roles in both WS and normal brain development and function. We used quantitative RT-PCR to determine the transcriptional level of 14 WS gene markers in a cohort of 77 persons with WS and 48 normal controls. Results reported here: (1) show that the expression of the genes deleted in WS is decreased in some but not all cases, (2) demonstrate that the parental origin of the deletion contributes to the level of expression of GTF2I independently of age and gender and (3) indicate that the correlation of expression between GTF2I and some other genes in the WS region differs in WS subjects and normal controls, which in turn points toward a regulatory role for this gene. Interspecies comparisons suggest GTF2I may play a key role in normal brain development. Journal of Human Genetics (2009) 54, 193-198; doi:10.1038/jhg.2009.5; published online 13 March 2009
\end{abstract}

Keywords: William's syndrome; gene expression; RT-PCR; parental origin; GTF2I

\section{INTRODUCTION}

William's syndrome (WS) is a neurogenetic disorder affecting human development and adult cognition. Caused by a microdeletion of chromosome band 7q11.23 involving $\sim 24-28$ genes and RNA transcripts, typical features of WS include dysmorphic craniofacial features (full lips, short nasal bridge and large forehead), infantile hypercalcemia, hypertension, supravalvular aortic stenosis and mental retardation; ${ }^{1,2}$ the latter four are important models of other common diseases. WS subjects exhibit striking peaks and valleys in neurocognition. There are deficits in visual-spatial and global processing whereas particular aspects of language expression and facial recognition are relative strengths; these features have recently been linked to variations in adult brain structure and function. ${ }^{3-5}$ WS subjects also exhibit a heightened drive to sociability. The physical and cognitive features associated with WS result in part from loss of one genomic copy of the deleted region. Other mechanisms contribute, including the effect of the deletion rearrangement on genes flanking the break point, and variations of DNA sequence, epigenetic mechanisms including imprinting, parent-of-origin and tissue-specific effects, all of which may alter the expression of genes located on the non-deleted chromosome 7. Because of these other factors regulating and maintaining transcription levels, ${ }^{6-9}$ deletion of one allele does not necessarily mean that gene expression will be half normal or even significantly decreased.
As an approach toward understanding the role of the deleted genes in WS, we have characterized WS subjects according to genetic, social/ emotional, neurocognitive, neurophysiological and neuroanatomical features. Previous work from this laboratory also used molecular cytogenetic, microsatellite and DNA sequencing analyses to characterize the structure and variation of the WS deletion in a cohort of 83 families. Testing of the hypothesis that differences in cognitive phenotypes would be in part related to differences in the genes deleted led to the discovery that lack of deletion of CYLN2, GTF2I, GTF2IRD1 alone, ${ }^{10}$ and recently, GTF2IRD1, was associated with relative preservation of a subset of visual-spatial functions. ${ }^{11,12}$ We next hypothesized that mechanisms other than the deletion itself may affect the expression level of WS-region genes. In contrast to the previous lack of consistent evidence for imprinted gene expression ${ }^{13,14}$ in this region, we found that hypertension was predicted by the parental origin of the deletion. ${ }^{15}$ We and others have proposed that gene expression, physical and possibly cognitive phenotypes in WS, are related to the parental origin of the deletion. ${ }^{13}$

This report addresses the hypotheses that: (1) expression of deleted genes in WS is related to parental origin as well as to the deletion itself, and (2) expression of genes within the WS region is correlated. We also suggest that variations of gene expression in WS contribute to WS features: full scale IQ, visual-spatial deficits, and social/emotional, linguistic and neuroanatomic variation.

${ }^{1}$ Division of Neurogenetics, Cedars-Sinai Medical Center and Departments of Human Genetics and Pediatrics, UCLA, Los Angeles, CA, USA; ${ }^{2}$ Department of Psychology, Emory University, Atlanta, GA, USA; ${ }^{3}$ Department of Neurology, Beth Israel Deaconess Medical Center, Boston, MA, USA; ${ }^{4}$ Department of Psychiatry and Behavioral Sciences, Stanford University School of Medicine, Palo Alto, CA, USA; ${ }^{5}$ Laboratory of Cognitive Neuroscience, Salk Institute for Biological Studies, La Jolla, CA, USA and ${ }^{6}$ The Brain Institute, University of Utah, Salt Lake City, UT, USA

Correspondence: Dr JR Korenberg, The Brain Institute, University of Utah, 383 Colorow Drive, Salt Lake City, UT 84108, USA.

E-mail: julie.korenberg@hsc.utah.edu

Received 20 August 2008; revised 4 December 2008; accepted 17 December 2008; published online 13 March 2009 


\section{MATERIALS AND METHODS}

\section{Subjects}

The population of this study includes a total of 77 William's syndrome subjects and 48 normal control subjects. The study was approved by the Internal Review Board of Cedars-Sinai Medical Center. All patients and their families gave written informed consent to participate in this study. The gender, age and number of subjects are detailed in Table 1.

\section{Determination of deletions}

Deletions in WS subjects were determined as previously described. ${ }^{16}$ To refine the deletion region and identify variability in deletion size, 12 microsatellite markers flanking ELN were typed in 77 WS patients and their parents. ${ }^{15}$ These highly polymorphic repeat markers were used in a multiplex polymerase chain reaction (PCR) to detect deletions and to determine the parental origins of deletions when present. All markers except ELN, ${ }^{17}$ D7S1870 ${ }^{18}$ and D7S489 19 were identified through the genome database and/or CEPH. PCR was carried out using $40 \mathrm{ng}$ of genomic DNA under standard conditions using either [ $\left.{ }^{32} \mathrm{P}\right]$ dCTP or fluorescently labeled primers, and $0.5 \mathrm{U}$ Taq polymerase. The PCR products were visualized by autoradiography following electrophoresis in $6 \%$ denaturing polyacrylamide gel or by ABI PRISM Genotyper 2.0 software run on an ABI 377 Sequence Detection System (Applied Biosystems Inc., Foster City, CA, USA).

\section{Quantification of gene expression}

To quantify relative gene expression, we generated and cultivated immortalized lymphoblast (LB) cell lines from each of 77 subjects and 48 normal controls, a subset of the parents who transmitted the normal chromosome 7. These cells were grown in RPMI-1640 with 10\% FBS, 5\% penicillin/streptomycin, $5 \%$ L-glutamine and $0.5 \%$ gentamicin. After RNA isolation from each of the 122 cell lines, cDNA libraries were synthesized, followed by quantitative real-time PCR using TaqMan Gene Expression Assays. Relative transcription was evaluated in each of the 122 samples (three controls did not grow well), for 14 genes that were expressed in LB cell lines located in the WS-deleted region.

\section{Preparation of cDNA libraries}

We isolated total RNA (Versagene RNA kit, Gentra Systems, Minneapolis, MN, USA). Cell suspensions were pelleted by centrifugation, washed with HBSS and homogenized in lysis buffer for $30 \mathrm{~s}$ with a Brinkmann PT 10/35 Homogenizer. RNA was isolated according to the kit protocol including DNase treatment. The RNA was evaluated by $1 \%$ RNase-free agarose gel electrophoresis, using a Nanodrop ND-1000 spectrophotometer (Nanodrop Technologies, Wilmington, DE, USA), and Agilent 2100 Bioanalyzer (24 samples, average rRNA 28S/18S was $2.0 \pm 0.2$ ). A260/A280 ratios measured were between 1.8 and 2.1. The samples were stored at $-80^{\circ} \mathrm{C}$.

cDNA libraries were prepared from total RNA using M-MLV Reverse Transcriptase (Invitrogen, Carlsbad, CA, USA) in an RNase/DNase-free $250 \mu \mathrm{l}$ reaction vessel containing $4 \mu \mathrm{g}$ total RNA, $1 \mu \mathrm{l}$ oligo(dT)15 $\left(500 \mu \mathrm{g} \mathrm{ml}^{-1}\right), 1 \mu \mathrm{l} 10 \mathrm{~mm}$ dNTP mix (10 mm each dATP, dGTP, dCTP and dTTP at neutral $\mathrm{pH}$ ) and sterile, distilled water to $20 \mu \mathrm{l}$. The mixture was heated to $65^{\circ} \mathrm{C}$ for $5 \mathrm{~min}$ and chilled on ice followed by addition of $4 \mu \mathrm{l}$ $5 \times$ first-strand buffer ( $250 \mathrm{~mm}$ Tris- $\mathrm{HCl}$ [pH 8.3], $375 \mathrm{~mm} \mathrm{KCl,} 15 \mathrm{~mm} \mathrm{MgCl}$ ),

Table 1 Number and age ranges of William's syndrome and normal control population

\begin{tabular}{lcc}
\hline Gender & Age (years) & Number \\
\hline $\begin{array}{lll}\text { William's syndrome } \\
\text { Male }\end{array}$ & 33 \\
Female & $24.6 \pm 10.5$ & 44 \\
Normal controls & $23.4 \pm 10.4$ & \\
$\quad$ Male & & 24 \\
Female & $54.3 \pm 12.3$ & 24 \\
\hline
\end{tabular}

$2 \mu \mathrm{l} \quad 0.1 \mathrm{M}$ DTT and $1 \mu \mathrm{l}$ RNase Recombinant Ribonuclease Inhibitor $\left(40 \mathrm{U}^{-1} \mathrm{l}^{-1}\right.$. This mixture was incubated at $37^{\circ} \mathrm{C}$ for $2 \mathrm{~min}$, followed by incubation at $37^{\circ} \mathrm{C}$ for $50 \mathrm{~min}$ and inactivation by heating at $70^{\circ} \mathrm{C}$ for $15 \mathrm{~min}$.

\section{Quantitative real-time PCR}

Quantitative real-time PCR measures the increase in fluorescence intensity over repeated PCR cycles, with the cycle number at which fluorescence becomes detectable being identified as the $C_{\mathrm{t}}$ value. The difference between the $C_{\mathrm{t}}$ of the target gene and a control gene is the $\Delta C_{\mathrm{t}}$ and reflects the relative decreased expression of the WS gene.

TaqMan Gene Expression Assays designed for human gene transcripts (Applied Biosystems, Foster City, CA, USA) were used for quantifying gene expression. The assay mixture consisted of a TaqMan MGB probe (labeled with FAM dye) and unlabeled PCR primers for a specific human gene. The fourteen genes and corresponding gene expression assays used in this study are listed in Supplementary Table 1. All of these map to chromosome band 7q11.23 as shown in Figure 1a. Of these, 12 are typically deleted in WS, and two are typically outside the deleted region. To optimize reproducibility, four replicates were used, the coefficient of variation was calculated for each set of three or four replicates and if it exceeded 5\%, the sample was excluded from statistical analysis. ${ }^{20}$

To ensure the unknown samples were run at a total mRNA concentration in the linear range of the assay, relative standard curves were established for each endogenous control and each gene of interest using five or four serial dilutions of pooled mRNA of known concentration (calculated from absorbance measurements; $0.15-1500 \mathrm{ng}^{-1} \mathrm{l}^{-1}$ for group 1, 26.67-0.0533 $\mathrm{ng}^{-1} \mathrm{l}^{-1}$ for group 2). Fluorescence threshold values were held constant across all plates/genes (User Bulletin No. 2, December 1997, Quantitative PCR; Applied Biosystems, Carlsbad, CA, USA).

\section{Analysis of gene expression}

Gene expression data were analyzed using the comparative $C_{\mathrm{t}}$ method. This method assumes approximately $100 \%$ PCR efficiency, which is achieved by the commercial TaqMan gene expression assays we employed. ABI showed that the comparative $C_{\mathrm{t}}$ method gave better replication values than did the standard curve method for TaqMan Gene Expression Assays and that larger dilution ranges decrease or minimize $C_{\mathrm{t}}$ variation due to a pipetting error (127AP05-02: Amplification Efficiency of TaqMan Gene Expression Assays: Application Note). Our comparative $C_{\mathrm{t}}$ method data yielded normal distributions, whereas our standard curve method data tended to have more Poisson-like distributions. Gene expression distributions were more normally distributed in the controls than in the two WS groups, which were similar. Distributions were evaluated with JMP 5.0: The Statistical Discovery Software (SAS Institute Inc., Cary, NC, USA).

Relative gene expression between the mean $C_{\mathrm{t}}$ of the WS population and the mean $C_{\mathrm{t}}$ of the control population was determined by the difference between the two populations. It is expressed as a function of 2 to the power of this difference.

\section{Statistical analysis}

Statistical significance of differences was evaluated using Student's $t$-test. Correlation coefficients were used to examine the correlation between the expression of specific gene pairs. Factors influencing gene expression in subjects with maternally or paternally derived deletions were determined using multiple regression analysis. All statistical analyses were two-tailed and performed with JMP 5.0: The Statistical Discovery Software (SAS Institute Inc., Cary, NC, USA).

\section{RESULTS}

To test the hypothesis that gene expression in WS is related to deletion, and hence to copy number, we compared each gene's mean $\beta$-actin (ACTB) normalized expression in WS subjects to that of normal controls; results are displayed in Figure 2. Decreased gene expression was found for nine of 12 typically deleted genes: FZD9, WSTF, STX1A, CLDN3, CLDN4, RFC2, CYLN2, WBSCR23, GTF2I $(P<0.0001$ for 

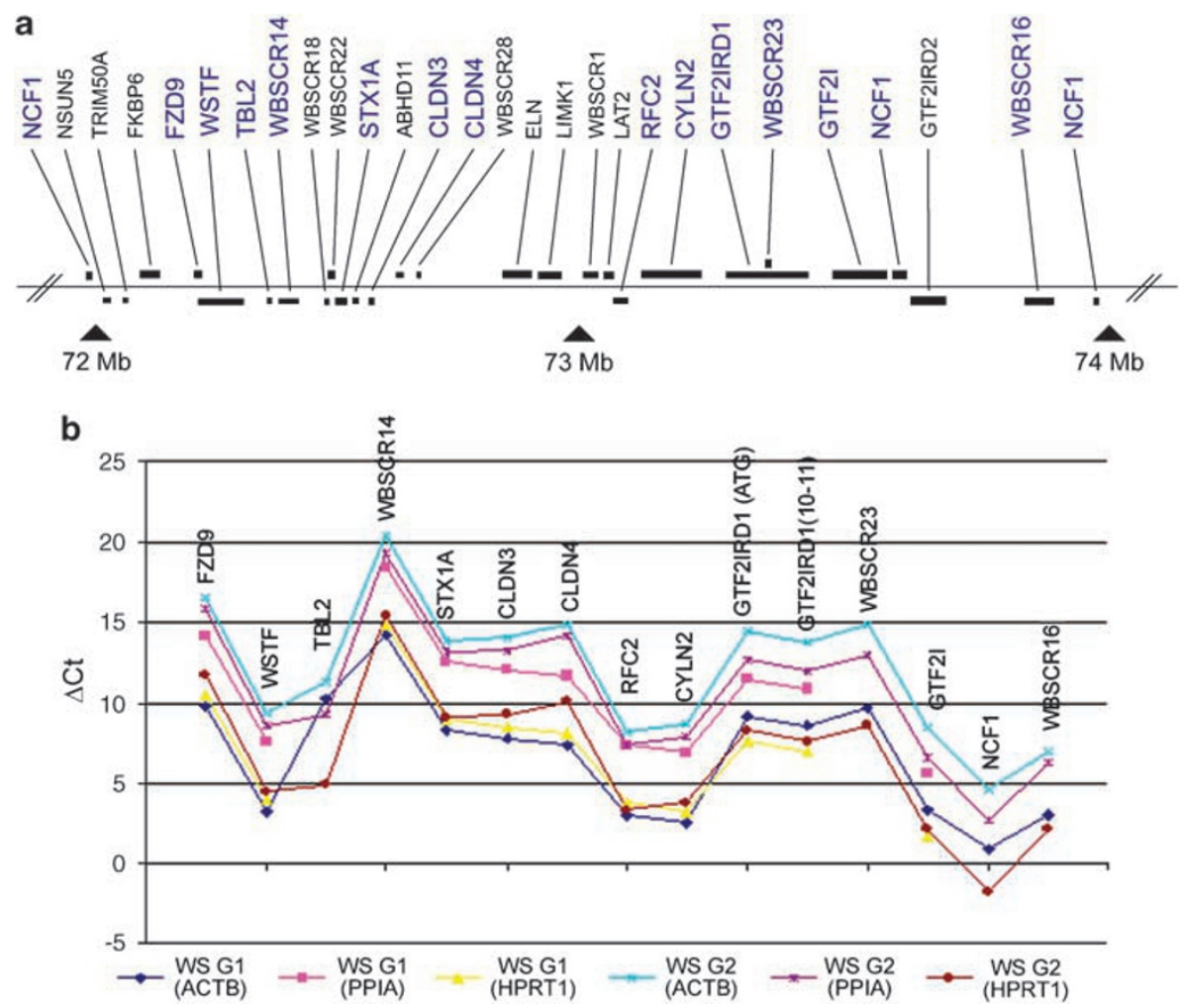

Figure 1 William's syndrome chromosome region genes and gene expression. (a) Map of genes commonly deleted in 7q11.23 (black) and analyzed in current report (blue); (b) Expression patterns over all 14 genes are similar for the two groups regardless of whether values are normalized relative to ACTB, PPIA or HPRT1. Data for these calculations are in Supplementary Table 2.

a
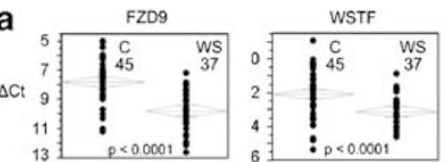

CYLN2

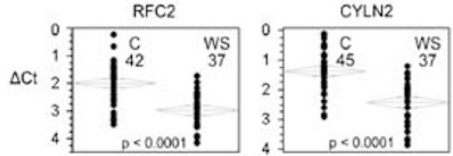

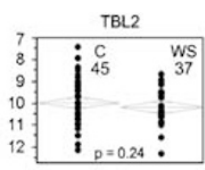
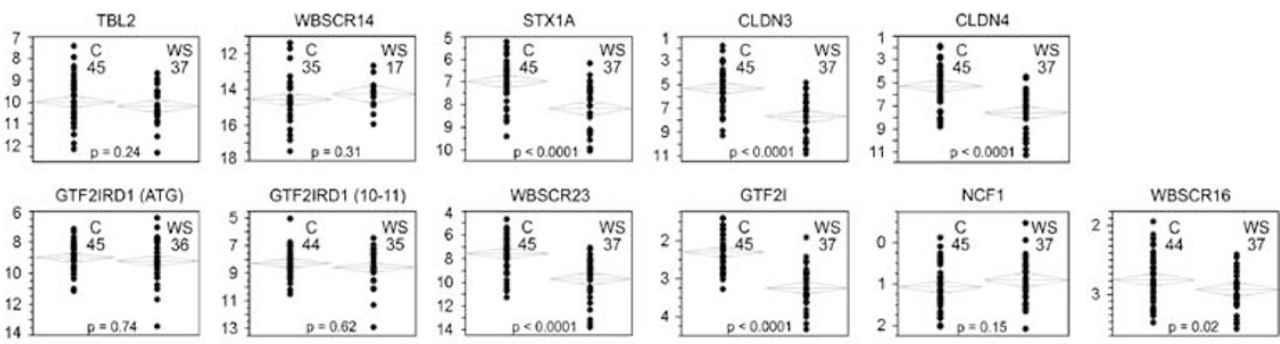

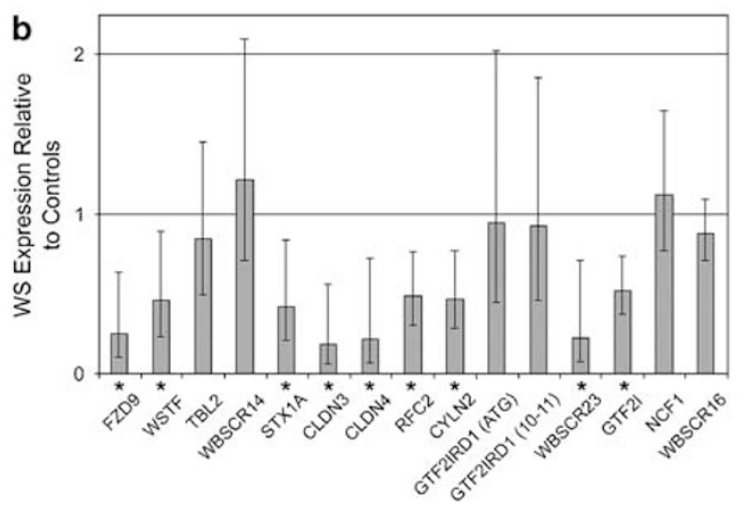

Figure 2 Quantitative expression of 14 genes in William's syndrome. Expression is decreased for many but not all deleted genes. (a) Plots of bivariate analysis for each gene; values for each normal control subject (left) and William's syndrome subjects (right) are normalized to ACTB and shown as $\Delta C_{t}$. Number of subjects in each group is also shown. (b) Bar graph showing each gene's median expression and s.d. (expression in normal controls $=1 ;^{*}=P<0.0001$ ). Data for these calculations are in Supplementary Table 1 . Error range is determined by evaluating the expression: $2-\Delta \Delta C_{\mathrm{t}}$ with $\Delta \Delta C_{\mathrm{t}}+\mathrm{s}$ and $\Delta \Delta C_{\mathrm{t}}-\mathrm{s}$, where $\mathrm{s}=$ the standard deviation of the $\Delta \Delta C_{\mathrm{t}}$ value according to User Bulletin No. 2: ABI Prism 7700 Sequence Detection System, December 1997 (Applied Biosystems). 
all). In contrast, there was no decrease for three other typically deleted genes: TBL2, WBSCR14 and GTF2IRD1, corroborated by studies of 8 WS. ${ }^{9}$ Data for this figure are in Supplementary Table 2.

To test the hypothesis that gene expression in WS was related to the parental origin of the deletion, we employed a two-group strategy with the groups determined by the date of receipt of the samples. In this strategy, multiple comparisons are done on an initial group to generate hypotheses. Hypotheses thus generated are then explicitly tested in a second, independent group. Hypotheses are considered to be supported only if they are statistically significant in the second group; potential hypotheses not supported in the initial group are permanently discarded and not tested further.

The first group consisted of 37 individuals with WS in whom we had previously determined the deletion and its parental origin. The gender, parental origin of deletion, age and number of subjects in each category are listed in Table 2 . Differences in $\Delta C_{t}$ between subjects with deletions on the maternally and paternally derived chromosomes were determined for 14 genes.

No statistically significant differences were found, although the expression of GTF2I was relatively lower $(P=0.13)$ in individuals with maternally derived deletions. We then tested the hypothesis that GTF2I is expressed at a lower level when it is on the paternally derived chromosome, in a second, independently identified and analyzed, group of 40 WS subjects. The tested hypothesis was statistically significant at $P=0.002$. Thus, the two-group approach demonstrates that the expression of GTF2I is lower when the single remaining copy is located on the paternally derived than when it is on the maternally derived chromosome. Figure 3 shows the ACTBnormalized $\Delta C_{\mathrm{t}}$ values for GTF2IRD1, WBSCR23 and GTF2I tested in WS groups 1 and 2. Figure 1b illustrates that similar expression patterns were seen in each group. Data for these figures are in Supplementary Table 3.

We used multivariate regression to test the possibility that the apparent parent-of-origin difference in GTF2I expression was actually due to independent effects of age and gender (Supplementary Table 4). However, age and gender did not contribute to the variance in gene expression; parent-of-origin was the only significant independent variable $(P=0.0004)$.

To quantitatively estimate the difference in GTF2I expression between paternally and maternally derived chromosomes, we compared the relative expression of the gene in subjects with the two types of deletions. Under the assumption of $100 \%$ PCR efficiency, as required by the comparative $C_{\mathrm{t}}$ method, we estimated the expression from the paternal copy at $61-85 \%$ of that from the maternal copy.

Table 2 Summary of Williams subjects in groups 1, 2 and 1+2

\begin{tabular}{llllr}
\hline Group & Gender & Parental origin of deletion & Age (years) & Number \\
\hline 1 & Male & Paternal & $26.5 \pm 9.0$ & 8 \\
& \multirow{3}{*}{ Female } & Maternal & $27.5 \pm 9.9$ & 11 \\
& & Paternal & $22.6 \pm 7.7$ & 10 \\
& Male & Maternal & $27.0 \pm 7.1$ & 9 \\
& \multirow{3}{*}{ Female } & Paternal & $23.6 \pm 11.7$ & 5 \\
& & Maternal & $21.6 \pm 12.4$ & 10 \\
& Male & Paternal & $23.2 \pm 7.8$ & 11 \\
& & Maternal & $21.6 \pm 13.8$ & 17 \\
& \multirow{3}{*}{ Female } & Paternal & $24.4 \pm 9.5$ & 12 \\
& & Maternal & $24.7 \pm 11.3$ & 21 \\
& & Paternal & $23.3 \pm 7.5$ & 19 \\
& & Maternal & $23.4 \pm 12.3$ & 25 \\
\hline
\end{tabular}

We next asked whether the expression level of a given gene within the WS region might be correlated with that of other genes located within the region and whether these correlations differed between normal subjects and those with William's syndrome. The correlations for a single gene pair are shown in Figure $4 \mathrm{~d}$. The expression of claudin $3(\mathrm{CLDN} 3)$ is highly correlated $(r=0.95)$ with that of claudin 4 (CLDN4) in subjects with WS and in normal individuals. Located $\sim 60 \mathrm{~Kb}$ apart in a head to head orientation, the sequences of CLDN3 and CLDN4 ${ }^{21}$ were found to be only $55 \%$ identical (peptide) and $81 \%$ identical (nucleotide) (NCBI Entrez) compared using Blast 2.2.12 (NCBI).

We then extended the analysis to all gene pairs within the region. Figure 4 a displays the correlation coefficient for each gene pair in the controls on the $x$ axis and that for WS subjects on the $y$ axis. The relative levels of expression are generally similar in WS and normal subjects (Figure 1b). However, there is a set of genes whose correlations with GTF2I differ notably between WS and control subjects, including several where the correlation with GTF2I is positive in WS but negative in normal individuals. The positive and negative bar graphs in Figure $4 \mathrm{~b}$ support the same conclusion as does the proportion of gene correlations with an inverse sign shown in $4 \mathrm{c}$. These data suggest that GTF2I may be involved in the regulation of other genes in the WS region.

\section{DISCUSSION}

Our results show that the expression of some genes is significantly lower in a large cohort of WS versus in normal controls, whereas that of other genes is not significantly different. Thus, the level of expression for genes in this region reflects regulatory control mechanisms in addition to an effect of copy number. This study also found that the expression of GTF2I in WS is related to the parental origin of the transmitted allele, lower when of paternal origin, which supports an epigenetic control mechanism and the hypothesis that GTF2I is paternally imprinted, possibly related to the upstream CpG island. ${ }^{21}$ GTF2I is a regulator of transcription and acts through direct binding to DNA and, to chromatin HDACs. Imprinting of this gene has not previously been reported. However, the effects of parental origin on linear growth and head circumference that have been reported ${ }^{13}$ but not confirmed, may be related to partial imprinting such as suggested by the current results. It is notable that the subtle difference between the maternal and paternal levels of expression may also be related to the risk of hypertension seen in WS as well as to autism linked to this region. ${ }^{22}$ Although it will require sensitive detection below the level of current array technologies, it will be of interest to determine that the subtle differences found here are also present in the normal population and whether they are associated with DNA methylation or chromatin modifications in the WS and normal populations. GTF2I may be one of many genes located throughout in the genome, whose expression differs when inherited through the maternal versus paternal germ lines, a model for subtle modulation of phenotype by parental origin.

Combining our results with the reported increased levels of GTF2I in humans versus our closest evolutionary relative, the common chimpanzee, Pan troglodytes. ${ }^{23}$ We hypothesize that the level and regulation of GTF2I may be in part related to the differences in regional brain anatomy seen in WS and reflect a genetic pathway contributing to normal human brain development. This is supported in part by studies (Allen Brain Atlas, http://www.brain-map.org ${ }^{24}$ ) showing GTF2I expression in neurons in all layers of human visual cortex, and in the mouse brain, by the regional concentration of GTF2I, highest in a subset of regions that differ in WS brain ${ }^{4}$ cerebral 
cortex, hippocampus, retrohippocampus and amygdala, as well as lateral septum, olfactory bulb and cerebellar Purkinje cells. Genetic approaches including the use of rare WS with atypical deletions also suggest a role for GTF2I and GTF2IRD $1^{11,16}$ in visual-spatial functions that have been correlated with regional brain variations in WS and possibly normal brain function. ${ }^{25}$ Finally, although other genes in the
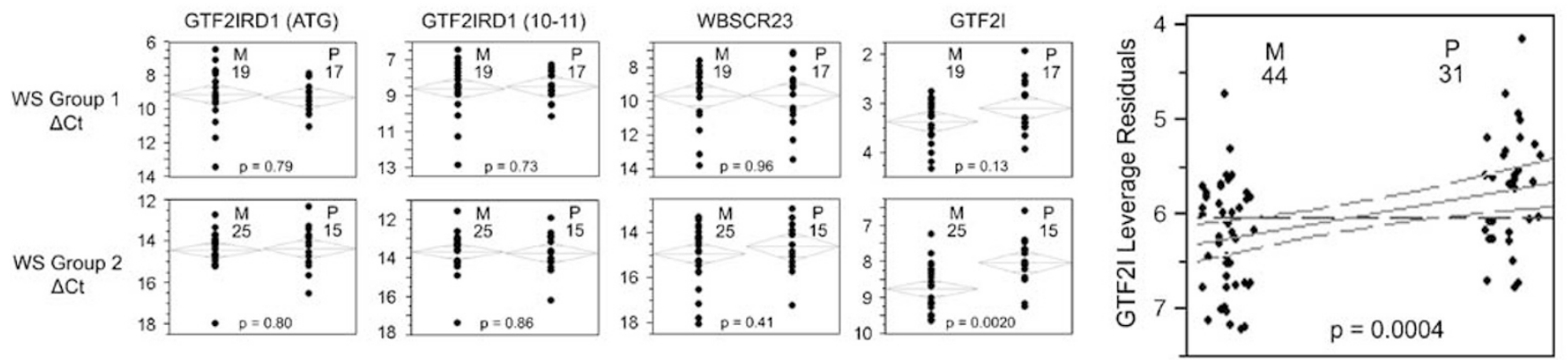

Figure 3 Effect of parental origin on gene expression in two independent groups of William's syndrome subjects. (a) In two independent groups, the expression of GTF2I, but not other selected genes, is lower in subjects with deletions on the maternal chromosome. Left panels show bivariate analysis for each gene in Group 1 (top) and Group 2 (bottom); number of subjects where the deleted chromosome of maternal (M) or paternal (P) is also displayed. Right panel shows leveraged residuals for GTF2I in the combined groups.
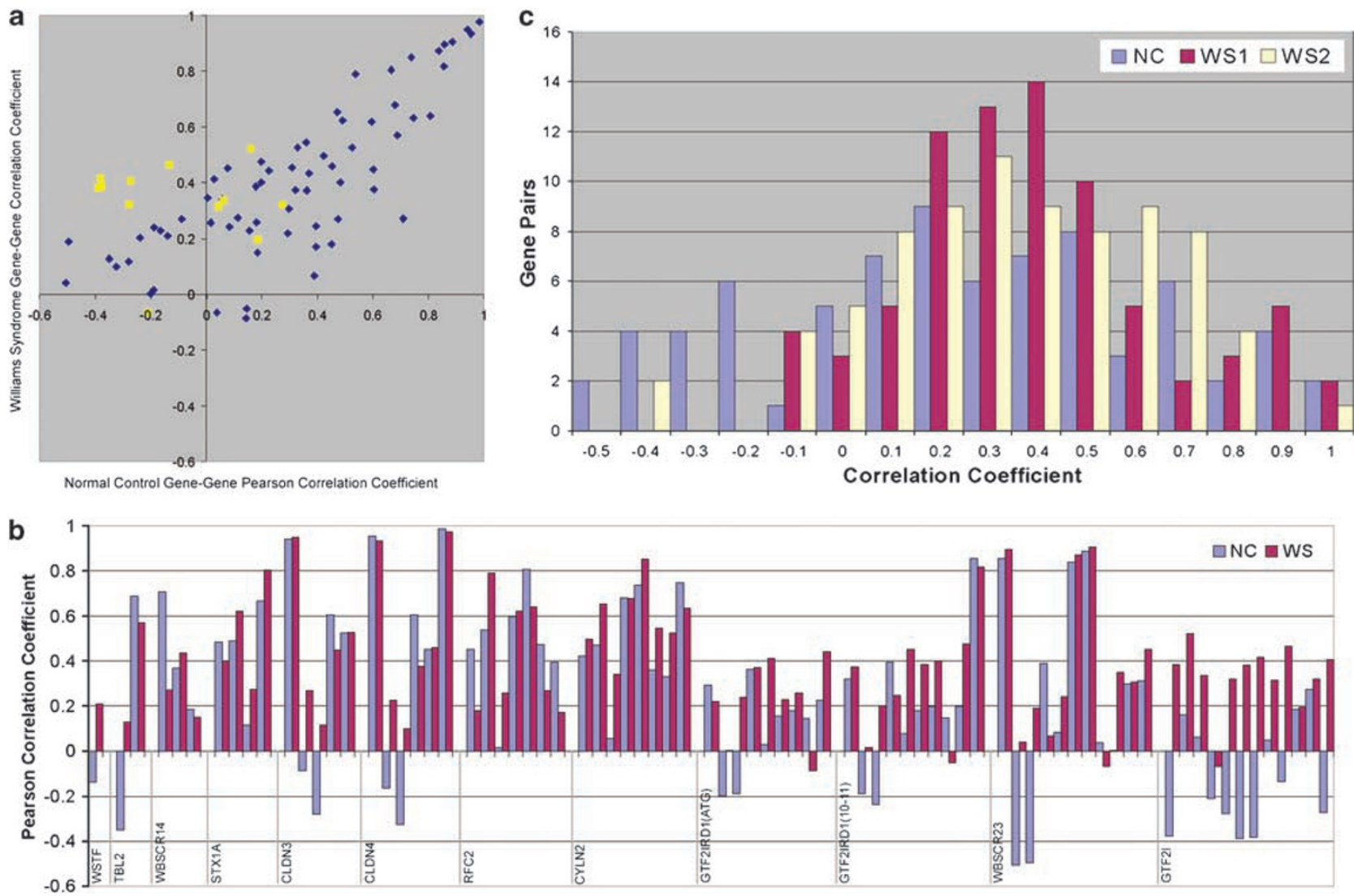

Figure 4 Pairwise correlation of expression of WS-deleted-region genes in WS subjects and normal controls. Correlation of GTF2I expression with that of other genes $(\boldsymbol{\square})$ reveals a set that are significantly positive in WS and significantly negative in normal controls. (a) Pearson correlation coefficients of gene pairs in normal controls ( $x$ axis) and William's syndrome group 1 ( $y$ axis); coefficients less than \pm 0.35 are not significant $(P>0.05$ ). (b) Quantitative pairwise gene to gene correlations used for $4 a$, displayed as bar graphs. These show that gene-to-gene correlations are similar in magnitude and direction in WS and NC except for GTF2I. The order of gene-to-gene correlations begins with the gene denoted at the left of the plot versus FZD9 then adds genes in genomic order from left to right. (for example, first comparison is WSTF versus FZD9, then TBL2 versus FZD9, TBL2 versus WSTF, followed by WBSCR14 versus FZD9, WBSCR14 versus WSTF, WBSCR14 versus TBL2, etc.). Note that among the largest differences in correlation coefficients between NC and WS are with GTF2I and FZD9, STX1A, CLDN3, CLDN4 and WBSCR23. These correlations change sign and are significant for both NC and WS. (c) Pairwise correlations of deleted WS genes in normal controls (NC), WS group 1 (WSG1) and WS Group 2 (WSG2). The degree of coexpression of CLDN3 and CLDN4 in NC, WSG1 and WSG2 is notable compared to other neighboring genes on chromosome 7 that are oriented head to head and located 50-70 kb apart as shown in Supplementary Figure 1. 
current study are also expressed in brain and likely also contribute to WS, neither their differential distributions nor studies of atypical deletions clearly implicate them in region-specific brain functions.

Patterns of imprinting/methylation, and thus epigenetic control, may vary between brain and LB. ${ }^{26}$ However, our use of quantitative RT-PCR in LB from a large number of WS, allows us to focus on particular gene interactions that are below the level of detection by chip expression arrays and represents a first step toward understanding the mechanisms underlying gene expression in the WS region. Although some tissue-specific variation in WS genes may be expected, ${ }^{9,27}$ recent data ${ }^{28}$ suggest commonalities among tissues. By allowing cross-tissue correlation of gene-to-gene expression patterns for a very large number of genes, chip/array data ${ }^{28}$ have revealed conservation of expression patterns across numerous tissues and evolution which supports the use of LB's for linking to some WS phenotypes. Further, the recent report showing common cellular mechanisms for synaptic vesicle fusion in neurons and non-neural intracellular fusion reactions ${ }^{27}$ support the likelihood that, for phenotypes due to these and other common cellular processes, part of our results for LB will also apply to the brain. We hypothesize that the expression mechanisms reported here will reflect a subset of those in developing adult brains and that LB may be useful to model subsets of genes and gene interactions in other tissues.

We conclude that decreased gene expression in a given tissue may contribute disproportionately to phenotype, and that subtle epigenetic effects on single genes or clusters of genes may contribute significantly to cognitive phenotypes. This subtle level of variation would not be detected by most current commercially available chip technologies. Furthermore, genes in the WS region may regulate neighboring gene expression and contribute to phenotype by multiple mechanisms. Specifically, we find that GTF2I, which, from interspecies comparisons may play a key role in normal human brain development, is both paternally imprinted and appears to regulate the expression of other genes in the WS region.

Future and ongoing experiments to elucidate the role of other WS genes in WS features include evaluating expression in brain and development, distinguishing differential allelic and monoallelic gene expression, analyzing the methylation and epigenetic control of genes in the WS region, and correlating quantitative gene expression to IQ, 'social/cognitive phenotypes and neuroanatomical variability.

\section{ACKNOWLEDGEMENTS}

The research presented in this article was supported by NICHD Grant HD 033113 and the James S McDonnell Foundation. JRK held the Geri and Richard Brawerman Chair in Molecular Genetics and is a USTAR Professor at the Brain Institute. We are grateful to the families who have donated the William's and control lymphoblast cell lines and to the local, regional, and national William's syndrome Associations.

1 Beuren, A. Supravalvular aortic stenosis: a complex syndrome with and without mental retardation. Birth Defects Orig. Art. Ser. VIII (5): 45-56 (1972).

2 Morris, C. A., Demsey, S. A., Leonard, C. O., Dilts, C. \& Blackburn, B. L. Natural history of Williams syndrome: physical characteristics. J. Pediatr. 113, 318-326 (1988).

3 Mills, D. L., Alvarez, T. D., St George, M., Appelbaum, L. G., Bellugi, U. \& Neville, H. III. Electrophysiological studies of face processing in Williams syndrome. J. Cogn. Neurosci. 12(Suppl 1), 47-64 (2000).
4 Reiss, A. L., Eckert, M. A., Rose, F. E., Karchemskiy, A., Kesler, S., Chang, M. et al. An experiment of nature: Brain anatomy parallels cognition and behavior in Williams syndrome. J. Neurosci. 24, 5009-5015 (2004).

5 Holinger, D. P., Bellugi, U., Mills, D. L., Korenberg, J. R., Reiss, A. L., Sherman, G. F. et al. Relative sparing of primary auditory cortex in Williams syndrome. Brain Res. 1037, 35-42 (2005).

6 Pastinen, T., Sladek, R., Gurd, S., Sammak, A., Ge, B. \& Lepage, P. et al. A survey of genetic and epigenetic variation affecting human gene expression. Physiol. Genomics 16, 184-193 (2004).

7 Robertson, K. D. DNA methylation and human disease. Nat. Rev. Genet. 6, 597-610 (2005).

8 Howald, C., Merla, G., Digilio, M. C., Amenta, S., Lyle, R., Deutsch, S. et al. Two high throughput technologies to detect segmental aneuploidies identify new Williams-Beuren syndrome patients with atypical deletions. J. Med. Genet. 43, 266-273 (2006).

9 Merla, G., Howald, C., Henrichsen, C. N., Lyle, R., Wyss, C., Zabot, M. T. et al. Submicroscopic deletion in patients with Williams-Beuren syndrome influences expression levels of the nonhemizygous flanking genes. Am. J. Hum. Genet. 79, 332-341 (2006).

10 Dai, L., Mills, D., Bellugi, U. \& Korenberg, J. R. Is it Williams Syndrome? GTF2IRD1 implicated in visual-spatial construction and GTF2I in sociability revealed by high resolution arrays. Am. J. Med. Genet. 149(A), 302-314 (2009).

11 Hirota, H., Matsuoka, R., Chen, X. N., Salandanan, L. S., Lincoln, A., Rose, F. E. et al. Williams syndrome deficits in visual spatial processing linked to GTF2IRD and GTF2I on chromosome 7q11.23. Genet. Med. 5, 311-321 (2003).

12 van Hagen, J. M., van der Geest, J. N., van der Giessen, R. S., Lagers-van Haselen, G. C., Eussen, H. J., Gille, J. J. et al. Contribution of CYLN2 and GTF2IRD1 to neurological and cognitive symptoms in Williams syndrome. Neurobiol. Dis. 26, 112-124 (2007)

13 Perez-Jurado, L. A., Peoples, R., Kaplan, P., Hamel, B. C. \& Francke, U. Molecular definition of the chromosome 7 deletion in Williams syndrome and parent-of-origin effects on growth. Am. J. Hum. Genet. 59, 781-792 (1996).

14 Wang, M. S., Schinzel, A., Kotzot, D., Balmer, D., Casey, R., Chodirker, B. N. et al. Molecular and clinical correlation study of Williams-Beuren syndrome: no evidence of molecular factors in the deletion region or imprinting affecting clinical outcome. Am. J. Med. Genet. 86, 34-43 (1999).

15 Antoine, H., Wijesuriya, H., Appelbaum, M., Chen, X. N., Simon, A., Rose, F. et al. Hypertension: Risk in Williams syndrome is determined by gender and parent of origin. Am. J. Hum. Genet 75(Suppl), A221 (2004).

16 Korenberg, J. R., Dai, L., Bellugi, U., Jarvinen-Pasley, A., Mills, D. L., Galaburda, A. et al. Deletion of 7q11.2 Genes and Williams Syndrome. In Inborn Errors of Development. The Molecular Basis of Clinical Disorders of Morphogenesis, 2nd edn. (eds. Epstein, C.J., Erickson, R.P., Wynshaw-Boris, A.) 1544-1555 (Oxford Univ. Press, New York, 2008).

17 Foster, K., Ferrell, R., King-Underwood, L., Povey, S., Attwood, J., Rennick, R. et al. Description of a dinucleotide repeat polymorphism in the human elastin gene and its use to confirm assignment of the gene to chromosome 7. Ann. Hum. Genet. 57, 87-96 (1993).

18 Gilbert-Dussardier, B., Bonneau, D., Gigarel, N., Le Merrer, M., Bonnet, D., Philip, N. et al. A novel microsatellite DNA marker at locus D7S1870 detects hemizygosity in $75 \%$ of patients with Williams syndrome. Am. J. Hum. Genet. 56, 542-544 (1995).

19 Robinson, W. P., Waslynka, J., Bernasconi, F., Wang, M., Clark, S., Kotzot, D. et al. Delineation of $7 q 11.2$ deletions associated with Williams-Beuren syndrome and mapping of a repetitive sequence to within and to either side of the common deletion. Genomics 34, 17-23 (1996).

20 Morello, F., de Bruin, T. W., Rotter, J. I., Pratt, R. E., van der Kallen, C. J., Hladik, G. A. et al. Differential gene expression of blood-derived cell lines in familial combined hyperlipidemia. Arterioscler Thromb Vasc. Biol. 24, 2149-2154 (2004).

21 Hillier, L. W., Fulton, R. S., Fulton, L. A., Graves, T. A., Pepin, K. H., WagnerMcPherson, C. et al. The DNA sequence of human chromosome 7. Nature 424, 157-164 (2003)

22 Ashley-Koch, A., Wolpert, C. M., Menold, M. M., Zaeem, L., Basu, S., Donnelly, S. L. et al. Genetic studies of autistic disorder and chromosome 7. Genomics 61, 227-236 (1999).

23 Caceres, M., Lachuer, J., Zapala, M. A., Redmond, J. C., Kudo, L. \& Geschwind, D. H. et al. Elevated gene expression levels distinguish human from non-human primate brains. Proc. Natl Acad. Sci. USA 100, 13030-13035 (2003).

24 Danoff, S. K., Taylor, H. E., Blackshaw, S. \& Desiderio, S. TFII-I, a candidate gene for Williams syndrome cognitive profile: parallels between regional expression in mouse brain and human phenotype. Neuroscience 123, 931-938 (2004).

25 Meyer-Lindenberg, A., Mervis, C. B. \& Berman, K. F. Neural mechanisms in Williams syndrome: a unique window to genetic influences on cognition and behaviour. Nature 7 , 380-393 (2006).

26 Esumi, S., Kakazu, N., Taguchi, Y., Hirayama, T., Sasaki, A., Hirabayashi, T. et al. Monoallelic yet combinatorial expression of variable exons of the protocadherin-alpha gene cluster in single neurons. Nat. Genet. 37, 171-176 (2005).

27 Jahn, R. \& Scheller, R. H. SNAREs-engines for membrane fusion. Nat. Rev. Mol. Cell Biol. 7, 631-643 (2006)

28 Chen, J., Blackwell, T. W., Fermin, D., Menon, R., Chen, Y., Gao, J. et al. Evolutionaryconserved gene expression response profiles across mammalian tissues. OMICS 11 , 96-115 (2007).

Supplementary Information accompanies the paper on Journal of Human Genetics website (http://www.nature.com/jhg) 